\title{
Conditioning and Dilation with Coherent Nearly-Linear Models
}

\author{
Renato Pelessoni $\left.{ }^{(}\right)(10$ and Paolo Vicig (1) \\ University of Trieste (DEAMS), 34123 Trieste, Italy \\ \{renato.pelessoni, paolo.vicig\}@deams.units.it
}

\begin{abstract}
In previous work [1] we introduced Nearly-Linear (NL) models, a class of neighbourhood models obtaining upper/lower probabilities by means of a linear affine transformation (with barriers) of a given probability. NL models are partitioned into more subfamilies, some of which are coherent. One, that of the Vertical Barrier Models (VBM), includes known models, such as the Pari-Mutuel, the $\varepsilon$-contamination or the Total Variation model as special instances. In this paper we study conditioning of coherent NL models, obtaining formulae for their natural extension. We show that VBMs are stable after conditioning, i.e. return a conditional model that is still a VBM, and that this is true also for the special instances mentioned above but not in general for NL models. We then analyse dilation for coherent NL models, a phenomenon that makes our ex-post opinion on an event $A$, after conditioning it on any event in a partition of hypotheses, vaguer than our ex-ante opinion on $A$.
\end{abstract}

Keywords: Conditioning · Coherent imprecise probabilities •

Nearly-Linear models · Dilation

\section{Introduction}

Among special imprecise probability models, neighbourhood models [10, Sec. 4.6.5] obtain an upper/lower probability from a given (precise) probability $P_{0}$. One reason for doing this may be that $P_{0}$ is not considered fully reliable. Even when it is, $P_{0}(A)$ represents a fair price for selling event $A$, meaning that the buyer is entitled to receive 1 if $A$ is true, 0 otherwise. A seller typically requires a higher price than $P_{0}(A), \bar{P}(A) \geq P_{0}(A)$, for selling $A$. The upper probability $\bar{P}$, to be interpreted as an infimum selling price in the behavioural approach to imprecise probabilities [10], is often obtained as a function of $P_{0}$.

Recently, we investigated Nearly-Linear (NL) models [1], a relatively simple class of neighbourhood models. In fact, they derive upper and lower probabilities, $\bar{P}$ and $\underline{P}$ respectively, as linear affine transformations of $P_{0}$ with barriers, to prevent reaching values outside the $[0,1]$ interval. As proven in [1], some NL models are coherent, while other ones ensure weaker properties. The most important coherent NL models are Vertical Barrier Models (VBM), including 
several well-known models as special cases, such as the Pari-Mutuel, the Total Variation, the $\varepsilon$-contamination model, and others.

In this paper we explore the behaviour of coherent NL models when conditioning. Precisely, after recalling essential preliminary notions in Sect.2, in Sect. 3 the events in the (unconditional) domain of $\underline{P}, \bar{P}$ are conditioned on an event $B$, and the lower/upper natural extensions $\underline{E}, \bar{E}$ of $\underline{P}, \bar{P}$ on the new (conditional) environment are computed. The natural extension is a standard inferential procedure in the theory of imprecise probabilities [10], which gives the least-committal coherent extension of a lower (upper) probability. Since $\underline{P}(\bar{P})$ is 2-monotone (2-alternating) in a coherent NL model, $\underline{E}(\bar{E})$ is given by easy-to-apply formulae and is 2-monotone (2-alternating) too. An interesting result is that VMBs are stable after conditioning: the conditional model after applying the natural extension is still a VBM. We show that this property extends also to the mentioned special VBMs: conditioning each special VBM model returns a special VBM model of the same kind. By contrast, the property does not hold for other NL models. In Sect. 4 we explore the phenomenon of dilation, where, given a partition of events $\mathcal{B}$, it happens for some event $A$ that $\underline{E}(A \mid B) \leq \underline{P}(A) \leq \bar{P}(A) \leq \bar{E}(A \mid B)$, for all $B \in \mathcal{B} \backslash\{\emptyset\}$. This means that our $a$ posteriori evaluations are vaguer than the a priori ones. We derive a number of conditions for dilation to occur or not to occur. Section 5 concludes the paper.

\section{Preliminaries}

In this paper we shall be concerned with coherent lower and upper probabilities, both conditional and unconditional. Coherent means in both cases Williamscoherent [11], in the structure-free version studied in [6]:

Definition 1. Let $\mathcal{D} \neq \varnothing$ be an arbitrary set of conditional events. A conditional lower probability $\underline{P}: \mathcal{D} \rightarrow \mathbb{R}$ is coherent on $\mathcal{D}$ iff $\forall n \in N, \forall s_{0}, s_{1}, \ldots, s_{n} \geq$ $0, \forall A_{0}\left|B_{0}, A_{1}\right| B_{1}, \ldots, A_{n} \mid B_{n} \in \mathcal{D}$, defining $\underline{G}=\sum_{i=1}^{n} s_{i} I_{B_{i}}\left(I_{A_{i}}-\underline{P}\left(A_{i} \mid B_{i}\right)\right)-$ $s_{0} I_{B_{0}}\left(I_{A_{0}}-\underline{P}\left(A_{0} \mid B_{0}\right)\right), B=\bigvee_{i=0}^{n} B_{i}$ (I $I_{A}$ indicator of event $\left.A\right)$, it holds that $\max \{\underline{G} \mid B\} \geq 0$.

A similar definition applies to upper probabilities. However, when considering simultaneously lower and upper probabilities, they will be conjugate, i.e.

$$
\underline{P}(A \mid B)=1-\bar{P}\left(A^{\mathrm{c}} \mid B\right) .
$$

Equation (1) lets us refer to lower (alternatively upper) probabilities only.

When $\mathcal{D}$ is made of unconditional events only, Definition 1 coincides with Walley's coherence [10]. In general, a (Williams-)coherent $\underline{P}$ on $\mathcal{D}$ has a coherent extension, not necessarily unique, on any set of conditional events $\mathcal{D}^{\prime} \supset \mathcal{D}$.

The natural extension $\underline{E}$ of $\underline{P}$ on $\mathcal{D}^{\prime}$ is the least-committal coherent extension of $\underline{P}$ to $\mathcal{D}^{\prime}$, meaning that if $\underline{Q}$ is a coherent extension of $\underline{P}$, then $\underline{E} \leq \underline{Q}$ on $\mathcal{D}^{\prime}$. Further, $\underline{E}=\underline{P}$ on $\mathcal{D}$ iff $\underline{P}$ is coherent $[6,10]$.

In this paper, we shall initially be concerned with unconditional lower probabilities $(\underline{P}(\cdot))$ and their conjugates $(\bar{P}(\cdot))$. 
Coherence implies that [10, Sec. 2.7.4]

$$
\begin{aligned}
& \text { if } A \Rightarrow B, \underline{P}(A) \leq \underline{P}(B), \bar{P}(A) \leq \bar{P}(B) \text { (monotonicity) } \\
& \underline{P}(A)+\bar{P}(B) \geq \underline{P}(A \vee B) .
\end{aligned}
$$

The domain $\mathcal{D}$ of $\underline{P}(\cdot), \bar{P}(\cdot)$ will often be $\mathcal{A}(\mathbb{P})$, the set of events logically dependent on a given partition $\mathbb{P}$ (the powerset of $\mathbb{P}$, in set theoretic language).

A lower probability $\underline{P}$, coherent on $\mathcal{A}(\mathbb{P})$, is 2-monotone if $\underline{P}(A \vee B) \geq$ $\underline{P}(A)+\underline{P}(B)-\underline{P}(A \wedge B), \forall A, B \in \mathcal{A}(\mathbb{P})$. Its conjugate $\bar{P}$ is 2-alternating, meaning that $\bar{P}(A \vee B) \leq \bar{P}(A)+\bar{P}(B)-\bar{P}(A \wedge B), \forall A, B \in \mathcal{A}(\mathbb{P})$.

2-monotone and 2-alternating coherent imprecise probabilities have some special properties [8-10]. In particular,

Proposition 1. ([9, Thm. 7.2],[10, Sec. 6.4.6]). If $\underline{P}$ is a coherent 2-monotone lower probability on $\mathcal{A}(\mathbb{P})$ and $\bar{P}$ is its conjugate, given $B \in \mathcal{A}(\mathbb{P})$ such that $\underline{P}(B)>0$, then, $\forall A \in \mathcal{A}(\mathbb{P})$,

$$
\begin{aligned}
& \bar{E}(A \mid B)=\frac{\bar{P}(A \wedge B)}{\bar{P}(A \wedge B)+\underline{P}\left(A^{\mathrm{c}} \wedge B\right)} \\
& \underline{E}(A \mid B)=\frac{\underline{P}(A \wedge B)}{\underline{P}(A \wedge B)+\bar{P}\left(A^{\circ} \wedge B\right)}
\end{aligned}
$$

$\underline{E}$ is 2-monotone $\overline{(E}$ is 2-alternating) on $\mathcal{A}(\mathbb{P}) \mid B=\{A \mid B: A \in \mathcal{A}(\mathbb{P})\}$, where $B$ is fixed. $\underline{E}, \bar{E}$ are conjugate.

\subsection{Nearly-Linear Models}

Nearly-Linear models have been defined in [1], where their basic properties have been investigated.

Definition 2. A Nearly-Linear Model is a couple $(\underline{P}, \bar{P})$ of conjugate lower and upper probabilities on $\mathcal{A}(\mathbb{P})$, where $\forall A \in \mathcal{A}(\mathbb{P}) \backslash\{\emptyset, \Omega\}$

$$
\begin{aligned}
& \underline{P}(A)=\min \left\{\max \left\{b P_{0}(A)+a, 0\right\}, 1\right\}, \\
& \bar{P}(A)=\max \left\{\min \left\{b P_{0}(A)+c, 1\right\}, 0\right\}
\end{aligned}
$$

and $\underline{P}(\emptyset)=\bar{P}(\emptyset)=0, \underline{P}(\Omega)=\bar{P}(\Omega)=1$.

In Eqs. (5), (6), $P_{0}$ is an assigned (precise) probability on $\mathcal{A}(\mathbb{P})$, while

$$
b>0, a \in \mathbb{R}, c=1-(a+b) .
$$

It has been shown in $[1$, Sec. 3.1$]$ that NL models are partitioned into three subfamilies, with varying consistency properties. Here we focus on the coherent NL models, which are all the models in the VBM subfamily and some of the HBM (to be recalled next), while, within the third subfamily, $\underline{P}$ and $\bar{P}$ are coherent iff the cardinality of $\mathbb{P}$ is 2 (therefore we neglect these latter models). 
Definition 3. A Vertical Barrier Model (VBM) is a NL model where (5), (6) specialise into

$$
\begin{gathered}
\underline{P}(A)=\max \left\{b P_{0}(A)+a, 0\right\}, \forall A \in \mathcal{A}(\mathbb{P}) \backslash\{\Omega\}, \underline{P}(\Omega)=1 \\
\bar{P}(A)=\min \left\{b P_{0}(A)+c, 1\right\}, \quad \forall A \in \mathcal{A}(\mathbb{P}) \backslash\{\emptyset\}, \quad \bar{P}(\emptyset)=0 \\
0 \leq a+b \leq 1, a \leq 0
\end{gathered}
$$

and $c$ is given by $(7)$ (hence $c \geq 0)$.

In a Horizontal Barrier Model $(H B M) \underline{P}, \bar{P}$ are given by Definition 2, hence by (5), (6), (7) $\forall A \in \mathcal{A}(\mathbb{P}) \backslash\{\emptyset, \Omega\}$, where $a, b$ satisfy the constraints

$$
a+b>1, b+2 a \leq 1
$$

(implying $a<0, b>1, c<0$ ).

Proposition 2. ([1]) $\underline{P}, \bar{P}$ are coherent and 2-monotone, respectively 2-alternating in any VBM; in a HBM they are so iff $\bar{P}$ is subadditive (i.e. $\bar{P}(A)+$ $\bar{P}(B) \geq \bar{P}(A \vee B), \forall A, B \in \mathcal{A}(\mathbb{P}))$.

Thus, VBMs and (partly) HBMs ensure very good consistency properties, while being relatively simple transformations of an assigned probability $P_{0}$. Further, a VBM generalises a number of well-known models. Among them we find:

- if $a+b=0$, the vacuous lower/upper probability model [10, Sec. 2.9.1]:

$$
\begin{aligned}
& \underline{P}_{V}(A)=0, \forall A \neq \Omega, \underline{P}_{V}(\Omega)=1, \\
& \bar{P}_{V}(A)=1, \forall A \neq \emptyset, \bar{P}_{V}(\emptyset)=0 ;
\end{aligned}
$$

- if $a=0,0<b<1$ (hence $c=1-b>0$ ), the $\varepsilon$-contamination model or linear-vacuous mixture model [10, Sec. 2.9.2], here $b=1-\varepsilon$ :

$$
\begin{aligned}
& \underline{P}_{\varepsilon}(A)=(1-\varepsilon) P_{0}(A), \forall A \neq \Omega, \underline{P}_{\varepsilon}(\Omega)=1, \\
& \bar{P}_{\varepsilon}(A)=(1-\varepsilon) P_{0}(A)+\varepsilon, \forall A \neq \emptyset, \underline{P}_{\varepsilon}(\emptyset)=0 ;
\end{aligned}
$$

- if $b=1+\delta>1, a=-\delta<0$ (hence $c=0$ ), the Pari-Mutuel Model [4,7], [10, Sec. 2.9.3]:

$$
\begin{aligned}
& \underline{P}_{P M M}(A)=\max \left\{(1+\delta) P_{0}(A)-\delta, 0\right\}, \\
& \bar{P}_{P M M}(A)=\min \left\{(1+\delta) P_{0}(A), 1\right\} ;
\end{aligned}
$$

- if $b=1,-1<a<0$ (hence $c=-a$ ), the Total Variation Model [2, Sec. 3], [7, Sec. 3.2]: ${ }^{1}$

$$
\begin{aligned}
& \underline{P}_{T V M}(A)=\max \left\{P_{0}(A)+a, 0\right\} \forall A \neq \Omega, \underline{P}_{T V M}(\Omega)=1, \\
& \bar{P}_{T V M}(A)=\min \left\{P_{0}(A)-a, 1\right\} \forall A \neq \emptyset, \bar{P}_{T V M}(\emptyset)=0 .
\end{aligned}
$$

\footnotetext{
${ }^{1}$ Note that $\underline{P}_{T V M}(A) \leq \bar{P}_{T V M}(A), \forall A$, since $a<0$.
} 


\section{Conditioning Coherent Nearly-Linear Models}

Given a coherent NL model $(\underline{P}, \bar{P})$ on $\mathcal{A}(\mathbb{P})$ and an event $B \in \mathcal{A}(\mathbb{P}) \backslash\{\emptyset\}$, assumed or known to be true, we look for the natural extensions $\underline{E}(A \mid B), \bar{E}(A \mid B)$ of $\underline{P}, \bar{P}$ respectively, for any $A \in \mathcal{A}(\mathbb{P})$. In other words, $\underline{P}, \bar{P}$ are extended on $\mathcal{A}(\mathbb{P}) \mid B$.

When $\underline{P}(B)=0$, we determine $\underline{E}, \bar{E}$ quickly thanks to Proposition 3, which follows after a preliminary Lemma, stated without proof in a finite setting in [7].

Lemma 1. Let $\underline{P}: \mathcal{D} \rightarrow \mathbb{R}$ be a coherent lower probability on $\mathcal{D}$, non-empty set of unconditional events, and $B \in \mathcal{D}, B \neq \emptyset$ such that $\underline{P}(B)=0$. Let also $\mathcal{S}=\left\{A_{i} \mid B\right\}_{i \in I}$ be a set of events such that $A_{i} \in \mathcal{D}, B \nRightarrow A_{i}, \forall i \in I$. Then, the lower probability $\underline{P}^{\prime}$ defined by

$$
\underline{P}^{\prime}(E)=\underline{P}(E), \forall E \in \mathcal{D}, \underline{P}^{\prime}\left(A_{i} \mid B\right)=0, \forall i \in I,
$$

is a coherent extension of $\underline{P}$ on $\mathcal{D} \cup\left\{A_{i} \mid B\right\}_{i \in I}$.

Proof. Firstly, since $A \wedge B \Rightarrow B$ and $\underline{P}(B)=0$, we can extend in a unique way $\underline{P}$ on $\mathcal{D} \cup\left\{A_{i} \wedge B\right\}_{i \in I}$ preserving coherence, letting $\underline{P}\left(A_{i} \wedge B\right)=0, \forall i \in I$.

To prove coherence of $\underline{P}^{\prime}$, take $E_{j} \in \mathcal{D}(j=1, \ldots, n), A_{k} \mid B \in \mathcal{S}(k=$ $1, \ldots, m)$ and $n+m$ real coefficients $s_{j}(j=1, \ldots, n), t_{k}(k=1, \ldots, m)$, such that at most one of them is negative, and define

$$
\underline{G}=\sum_{j=1}^{n} s_{j}\left(I_{E_{j}}-\underline{P}^{\prime}\left(E_{j}\right)\right)+\sum_{k=1}^{m} t_{k} I_{B}\left(I_{A_{k}}-\underline{P}^{\prime}\left(A_{k} \mid B\right)\right) .
$$

According to Definition 1, we have to prove that $\max \{\underline{G} \mid H\} \geq 0$, where $H=\Omega$ if $n>0, H=B$ otherwise. We distinguish two cases.

(a) Let $n=0$, hence $\underline{G}=\sum_{k=1}^{m} t_{k} I_{B} I_{A_{k}}$. If $t_{k} \geq 0, \forall k=1, \ldots, m, \underline{G} \geq 0$ and $\max \{\underline{G} \mid H\}=\max \{\underline{G} \mid B\} \geq 0$. Otherwise, if $t_{\bar{k}}<0$ and $t_{k} \geq 0, \forall k=$ $1, \ldots, m, k \neq \bar{k}$, then $\max \{\underline{G} \mid H\} \geq \max \left\{t_{\bar{k}} I_{B} I_{A_{\bar{k}}} \mid B\right\}=\max \left\{t_{\bar{k}} I_{A_{\bar{k}}} \mid B\right\}=0$, where the last equality holds because $A_{\bar{k}}^{\mathrm{c}} \wedge B \neq \emptyset$ (since $B \nRightarrow A_{\bar{k}}$ ).

(b) If $n>0, \underline{G}=\sum_{j=1}^{n} s_{j}\left(I_{E_{j}}-\underline{P}^{\prime}\left(E_{j}\right)\right)+\sum_{k=1}^{m} t_{k} I_{B} I_{A_{k}}=\sum_{j=1}^{n} s_{j}\left(I_{E_{j}}-\right.$ $\left.\underline{P}\left(E_{j}\right)\right)+\sum_{k=1}^{m} t_{k}\left(I_{A_{k} \wedge B}-\underline{P}\left(A_{k} \wedge B\right)\right)$. Then, $\max \{\underline{G} \mid H\}=\max \{\underline{G}\} \geq 0$, applying Definition 1 to the coherent extension of $\underline{P}$ on $\mathcal{D} \cup\left\{A_{i} \wedge B\right\}_{i \in I}$.

Proposition 3. Let $\underline{P}: \mathcal{D} \rightarrow \mathbb{R}$ be a coherent lower probability on $\mathcal{D}$, non-empty set of unconditional events, and $B \in \mathcal{D}, B \neq \emptyset$ such that $\underline{P}(B)=0$. Then the natural extension $\underline{E}$ of $\underline{P}$ on $\mathcal{D} \cup\left\{A_{i} \mid B\right\}_{i \in I}$, where $A_{i} \in \mathcal{D}, \forall i \in I$, is given by $\underline{E}(F)=\underline{P}(F), \forall F \in \mathcal{D}$ and, $\forall i \in I$, by

$$
\underline{E}\left(A_{i} \mid B\right)=1 \text { if } B \Rightarrow A_{i}, \underline{E}\left(A_{i} \mid B\right)=0 \text { otherwise. }
$$

Proof. Since $\underline{P}$ is coherent on $\mathcal{D}$, we have $\underline{E}(F)=\underline{P}(F), \forall F \in \mathcal{D}$.

Let $j \in I$. If $B \Rightarrow A_{j}, A_{j}|B=B| B$, hence coherence of $\underline{E}$ implies $\underline{E}\left(A_{j} \mid B\right)=$ 1. If $B \nRightarrow A_{j}$, by Lemma $1, \underline{P}$ can be extended on $A_{j} \mid B$, letting $\underline{P}\left(A_{j} \mid B\right)=0$. Since $\underline{E}$ is the least-committal coherent extension of $\underline{P}$, we get $0 \leq \underline{E}\left(A_{j} \mid B\right) \leq$ $\underline{P}\left(A_{j} \mid B\right)=0$, hence $\underline{E}\left(A_{j} \mid B\right)=0$. 
Proposition 3 ensures that:

- $\underline{E}(A \mid B)=0$ if $B \nRightarrow A, \underline{E}(A \mid B)=1$ if $B \Rightarrow A$ (just take $A_{i}=A$ in (14));

- $\overline{\bar{E}}(A \mid B)=1$ if $B \nRightarrow A^{\mathrm{c}}, \bar{E}(A \mid B)=0$ if $B \Rightarrow A^{\mathrm{c}}$ (just take $A_{i}=A^{\mathrm{c}}$ in (14) and apply conjugacy).

Let us now assume $\underline{P}(B)>0$.

Then, $\underline{E}, \bar{E}$ are given by the next

Proposition 4. Let $(\underline{P}, \bar{P})$ be a coherent $N L$ model on $\mathcal{A}(\mathbb{P})$. For a given $B \in$ $\mathcal{A}(\mathbb{P})$ such that $\underline{P}(B)>0$, we have that

$$
\begin{aligned}
& \bar{E}(A \mid B)=\left\{\begin{array}{l}
0 \text { iff } \bar{P}(A \wedge B)=0 \\
\frac{b P_{0}(A \wedge B)+c}{b P_{0}(B)+1-b} \\
1 \text { iff } \underline{P}\left(A^{\mathrm{c}} \wedge B\right)=0
\end{array}(\in] 0,1[) \text { iff } \underline{P}\left(A^{\mathrm{c}} \wedge B\right), \bar{P}(A \wedge B) \in\right] 0,1[ \\
& \underline{E}(A \mid B)=\left\{\begin{array}{l}
1 \text { iff } \bar{P}\left(A^{\mathrm{c}} \wedge B\right)=0 \\
\frac{b P_{0}(A \wedge B)+a}{b P_{0}(B)+1-b} \\
0 \text { iff } \underline{P}(A \wedge B)=0
\end{array}(\in] 0,1[) \text { iff } \underline{P}(A \wedge B), \bar{P}\left(A^{\mathrm{c}} \wedge B\right) \in\right] 0,1[
\end{aligned}
$$

Proof. We derive first the expression (15) for $\bar{E}(A \mid B)$.

Since $\bar{P}$ is 2-alternating, we may apply Eq. (3). There, $\bar{E}$ depends on $\bar{P}(A \wedge B)$ and $\underline{P}\left(A^{\mathrm{c}} \wedge B\right)$, which cannot be simultaneously 0 : by $(2)$, this would imply $0=\underline{P}\left(A^{\mathrm{c}} \wedge B\right)+\bar{P}(A \wedge B) \geq \underline{P}(B)$, hence $\underline{P}(B)=0$. Further,

$$
\begin{aligned}
& \underline{P}\left(A^{\mathrm{c}} \wedge B\right)=1 \rightarrow \bar{P}\left(A \vee B^{\mathrm{c}}\right)=0 \rightarrow \bar{P}(A \wedge B)=0, \\
& \bar{P}(A \wedge B)=1 \rightarrow \underline{P}\left(A^{\mathrm{c}} \vee B^{\mathrm{c}}\right)=0 \rightarrow \underline{P}\left(A^{\mathrm{c}} \wedge B\right)=0,
\end{aligned}
$$

using in both derivations conjugacy first, monotonicity then.

Thus, only the following exhaustive alternatives may occur:

(a) $\bar{P}(A \wedge B)=0$

(b) $\underline{P}\left(A^{\mathrm{c}} \wedge B\right)=0$

(c) $\left.\underline{P}\left(A^{\mathrm{c}} \wedge B\right), \bar{P}(A \wedge B) \in\right] 0,1[$.

It is immediate from (3) that $\bar{E}(A \mid B)=0$ iff $\bar{P}(A \wedge B)=0$ and that $\bar{E}(A \mid B)=1$ iff $\underline{P}\left(A^{\mathrm{c}} \wedge B\right)=0$. Otherwise, $\left.\bar{E}(A \mid B) \in\right] 0,1[$. Precisely, from (3), (5), (6), (7)

$$
\bar{E}(A \mid B)=\frac{b P_{0}(A \wedge B)+c}{b P_{0}(A \wedge B)+c+b P_{0}\left(A^{\mathrm{c}} \wedge B\right)+a}=\frac{b P_{0}(A \wedge B)+c}{b P_{0}(B)+1-b} .
$$

Turning now to $\underline{E}(A \mid B)$, its value in Eq. (16) may be obtained simply by conjugacy, using $\underline{E}(A \mid B)=1-\bar{E}\left(A^{\mathrm{c}} \mid B\right)$ and (15). 
For the VBM, it is productive to write $\underline{E}(A \mid B), \bar{E}(A \mid B)$ as follows:

Proposition 5. Let $(\underline{P}, \bar{P})$ be a $V B M$ on $\mathcal{A}(\mathbb{P})$. For a given $B \in \mathcal{A}(\mathbb{P})$, with $\underline{P}(B)>0$, we have that

$$
\begin{gathered}
\underline{E}(A \mid B)=\max \left\{b_{B} P_{0}(A \mid B)+a_{B}, 0\right\}, \forall A \in \mathcal{A}(\mathbb{P}) \backslash\{\Omega\}, \underline{E}(\Omega \mid B)=1 \\
\bar{E}(A \mid B)=\min \left\{b_{B} P_{0}(A \mid B)+c_{B}, 1\right\}, \forall A \in \mathcal{A}(\mathbb{P}) \backslash\{\emptyset\}, \quad \bar{E}(\emptyset \mid B)=0 \\
a_{B}=\frac{a}{b P_{0}(B)+1-b}, b_{B}=\frac{b P_{0}(B)}{b P_{0}(B)+1-b}, c_{B}=1-\left(a_{B}+b_{B}\right) .
\end{gathered}
$$

Moreover, it holds that $b_{B}>0, a_{B} \leq 0,0<a_{B}+b_{B} \leq 1$.

Proof. Preliminarily, note that the denominator in (20) is positive. In fact, by assumption $\underline{P}(B)>0$, meaning by (8) that $b P_{0}(B)+a>0$. Using (7) and recalling that $c \geq 0$ in a $\mathrm{VBM}$, it holds also that

$$
b P_{0}(B)+1-b=b P_{0}(B)+a+c>0
$$

Given this, let us prove (18) (the argument for (19) is analogous or could be also derived using conjugacy and will be omitted). For this, recalling (16), it is sufficient to establish that

(i) $b_{B} P_{0}(A \mid B)+a_{B} \leq 1$, with equality holding iff $\bar{P}\left(A^{\mathrm{c}} \wedge B\right)=0$;

(ii) $b_{B} P_{0}(A \mid B)+a_{B} \leq 0$ iff $\underline{P}(A \wedge B)=0$;

(iii) $\left.b_{B} P_{0}(A \mid B)+a_{B}=\frac{b P_{0}(\bar{A} \wedge B)+a}{b P_{0}(B)+1-b} \in\right] 0,1\left[\right.$ iff $\left.\underline{P}(A \wedge B), \bar{P}\left(A^{\mathrm{c}} \wedge B\right) \in\right] 0,1[$.

(i) Using (20) and the product rule at the first equality, non-negativity of $b, c$ in the VBM at the inequality, (7) at the second equality, we obtain:

$$
\begin{aligned}
b_{B} P_{0}(A \mid B)+a_{B} & =\frac{b P_{0}(A \wedge B)+a}{b P_{0}(B)+1-b} \\
& \leq \frac{b P_{0}(A \wedge B)+b P_{0}\left(A^{\mathrm{c}} \wedge B\right)+a+c}{b P_{0}(B)+1-b}=1 .
\end{aligned}
$$

Moreover, $b_{B} P_{0}(A \mid B)+a_{B}=1$ iff $b P_{0}\left(A^{\mathrm{c}} \wedge B\right)+c=0$, which is equivalent to $\bar{P}\left(A^{\mathrm{c}} \wedge B\right)=0$ by $(9)$ (and since $b P_{0}\left(A^{\mathrm{c}} \wedge B\right)+c \geq 0$ ).

(ii) Taking account of the first equality in the proof of (i) above and since $b P_{0}(B)+1-b>0$, it ensues that

$$
b_{B} P_{0}(A \mid B)+a_{B} \leq 0 \text { iff } b P_{0}(A \wedge B)+a \leq 0 \text { iff } \underline{P}(A \wedge B)=0 .
$$

(iii) Immediate from (i), (ii) and recalling (17) (exchanging there $A$ with $A^{\mathrm{c}}$ ).

Elementary computations ensure that $b_{B}>0, a_{B} \leq 0,0<a_{B}+b_{B} \leq 1$. 
Proposition 5 proves an interesting feature of a VBM: when all events in $\mathcal{A}(\mathbb{P})$ are conditioned on the same $B$, the resulting model is still a VBM. (Note the this holds also when $\underline{P}(B)=0$ : here Proposition 5 does not apply, but from Proposition 3 we obtain the vacuous lower/upper probabilities, a special VBM.)

We synthesise this property saying that a VBM is stable under conditioning.

A natural question now arises: does a HBM ensure an analogous property? Specifically, condition on $B$, with $\underline{P}(B)>0$, the events of $\mathcal{A}(\mathbb{P})$, which are initially given a HBM lower probability $\underline{P}$. Is it the case that the resulting $\underline{E}(\cdot \mid B)$ is determined by the equation

$$
\underline{E}(A \mid B)=\min \left\{\max \left\{b_{B} P_{0}(A \mid B)+a_{B}, 0\right\}, 1\right\}
$$

with $a_{B}, b_{B}$ given by (20) and obeying the HBM constraints (11) (and similarly with $\bar{P}, \bar{E}(\cdot \mid B))$ ?

The answer is negative: although $\underline{E}(A \mid B)$ may occasionally be obtained from (21), for instance - as is easy to check - when $\left.\underline{P}(A \wedge B), \bar{P}\left(A^{\mathrm{c}} \wedge B\right) \in\right] 0,1[$, $\forall A \in \mathcal{A}(\mathbb{P}) \backslash\{\emptyset, \Omega\}$, this is not true in general, as shown in the next example.

Example 1. Given $\mathbb{P}=\left\{\omega_{1}, \omega_{2}, \omega_{3}\right\}$, Table 1 describes the values of an assigned $P_{0}$, and of $\underline{P}, \bar{P}$ obtained by (5), (6) with $a=-10, b=12$. Since $a, b$ satisfy (11) and, as can be easily checked, $\bar{P}$ is subadditive, $(\underline{P}, \bar{P})$ is a coherent HBM by Definition 3 and Proposition 2. Now, take $A=\omega_{2}, B=\omega_{1} \vee \omega_{2}$. From

Table 1. Data for Example 1

\begin{tabular}{l|l|l|l|l|l|l|l|l}
\hline & $\omega_{1}$ & $\omega_{2}$ & $\omega_{3}$ & $\omega_{1} \vee \omega_{2}$ & $\omega_{1} \vee \omega_{3}$ & $\omega_{2} \vee \omega_{3}$ & $\emptyset$ & $\Omega$ \\
\hline$P_{0}$ & $\frac{3}{10}$ & $\frac{3}{5}$ & $\frac{1}{10}$ & $\frac{9}{10}$ & $\frac{4}{10}$ & $\frac{7}{10}$ & 0 & 1 \\
\hline$\underline{P}$ & 0 & 0 & 0 & 0.8 & 0 & 0 & 0 & 1 \\
\hline $\bar{P}$ & 1 & 1 & 0.2 & 1 & 1 & 1 & 0 & 1 \\
\hline
\end{tabular}

(16), $\underline{E}(A \mid B)=0$, because $\underline{P}(A \wedge B)=\underline{P}\left(\omega_{2}\right)=0$. Yet, since $b_{B} P_{0}(A \mid B)+$ $a_{B}=\frac{b P_{0}\left(\omega_{2}\right)+a}{b P_{0}\left(\omega_{1} \vee \omega_{2}\right)+1-b}=14>1$, Eq. (21) would let us mistakenly conclude that $\underline{E}(A \mid B)=1$.

Thus, a coherent HBM differs from a VBM with respect to conditioning on some event $B$, being not stable.

It is interesting to note that not only the VBM, but also its special submodels listed in Sect. 2.1 are stable: conditioning one of them on $B$ returns a model of the same kind. Let us illustrate this in some detail.

For the linear-vacuous model $\left(\underline{P}_{V}, \bar{P}_{V}\right)$, it is well-known [10] that $\underline{E}_{V}(A \mid B)=$ 0 if $B \nRightarrow A$, while $\underline{E}_{V}(A \mid B)=1$ if $B \Rightarrow A$. Note that this follows also from Proposition 3, since $\underline{P}_{V}(B)=0$. By conjugacy, $\bar{E}_{V}(\cdot \mid B)$ is vacuous too.

With the $\varepsilon$-contamination model, its conditional model is again of the same type: from (18), (20), we get $\left.a_{B}=0, b_{B}=1-\varepsilon_{B} \in\right] 0,1[$. 
Turning to the Total Variation Model (TVM) and applying again (18), (20)

$$
\begin{aligned}
b_{B} & =1, a_{B}=\frac{a}{P_{0}(B)}<0 \\
\underline{E}_{T V M}(A \mid B) & =\max \left\{P_{0}(A \mid B)+\frac{a}{P_{0}(B)}, 0\right\} \\
& =\frac{1}{P_{0}(B)} \max \left\{P_{0}(A \wedge B)+a, 0\right\} .
\end{aligned}
$$

At first sight, the conditional model differs from a TVM. However, we may easily write (13) in the form (22):

$$
\underline{P}_{T V M}(A)=\frac{1}{P_{0}(\Omega)} \cdot \max \left\{P_{0}(A \wedge \Omega)+a, 0\right\} .
$$

Comparing (22) and (23) we see that the TVM is stable too under conditioning: $\underline{P}_{T V M}, \underline{E}_{T V M}$ may be thought of as normalised on the $P_{0}$-probability of what is currently assumed to be true ( $\Omega$ first, $B$ then).

The conjugate of $\underline{E}_{T V M}(A \mid B)$ is $\bar{E}_{T V M}(A \mid B)=\frac{1}{P_{0}(B)} \min \left\{P_{0}(A \wedge B)-a, 1\right\}$. $\underline{E}_{T V M}(A \mid B), \bar{E}_{T V M}(A \mid B)$ determine a credal set (i.e. the set of probabilities $P$ such that $\left.\underline{E}_{T V M}(A \mid B) \leq P(A \mid B) \leq \bar{E}_{T V M}(A \mid B), \forall A \in \mathcal{A}(\mathbb{P})\right)$ still made of all probabilities at a total variation distance ${ }^{2}$ from $P_{0}$ not larger than $-a(>0)$. This is like the unconditional TVM, the difference being that any $A$ is replaced by $A \wedge B$, i.e. by what remains possible of $A$ after assuming $B$ true.

Conditioning the Pari-Mutuel Model (PMM) on $B$ leads to similar conclusions: the conditional model is again of the PMM type.

Take for instance $\underline{P}_{P M M}(A)$, given by (12), where $b=1+\delta>1, a=-\delta<0$. From (18), (20) and recalling that $\underline{P}_{P M M}(B)=(1+\delta) P_{0}(B)-\delta>0$, we obtain

$$
\begin{aligned}
& a_{B}=\frac{-\delta}{\underline{P}_{P M M}(B)}<0, b_{B}=1+\frac{\delta}{\underline{P}_{P M M}(B)}>1 \\
& \underline{E}_{P M M}(A \mid B)=\max \left\{\left(1+\delta_{B}\right) P_{0}(A \mid B)-\delta_{B}, 0\right\}, \delta_{B}=\frac{\delta}{\underline{P}_{P M M}(B)} .
\end{aligned}
$$

Clearly, $a_{B}+b_{B}=1$, and we may conclude that $\underline{E}_{P M M}(\cdot \mid B)$ is again a PMM, with $\delta$ replaced by $\delta_{B}$. Note that the starting $\underline{P}_{P M M}(\cdot)$ may be written as

$$
\underline{P}_{P M M}(A)=\max \left\{(1+\delta) P_{0}(A \mid \Omega)-\frac{\delta}{\underline{P}_{P M M}(\Omega)}, 0\right\} .
$$

Similarly, we obtain

$$
\bar{E}_{P M M}(A \mid B)=\min \left\{\left(1+\delta_{B}\right) P_{0}(A \mid B), 1\right\} .
$$

Note that $\delta_{B} \geq \delta$, with equality holding iff $P_{0}(B)=1$. As well known $[7,10]$, $\delta$ (hence $\delta_{B}$ ) has the interpretation of a loading factor, which makes a subject

\footnotetext{
2 On the total variation distance see e.g. [3, Sec. 4.1].
} 
'sell' $A(A \mid B)$ for a selling price $\bar{P}_{P M M}(A)\left(\bar{E}_{P M M}(A \mid B)\right)$ higher than the 'fair price' $P_{0}(A)\left(P_{0}(A \mid B)\right)$. With respect to this kind of considerations, conditioning increases the loading factor, and the smaller $\underline{P}_{P M M}(B)$, the higher the increase. Next to this, conditioning makes both the seller and the buyer more cautious, in the sense that they restrict the events they would sell or buy.

From the seller's perspective, we can see this noting that $\bar{P}_{P M M}(A)<1$ iff $P_{0}(A)<\frac{1}{1+\delta}=\bar{t}_{\Omega}$. Here $\bar{t}_{\Omega}$ is the threshold to ensure that selling $A$ may be considered: when $\bar{P}(A)=1$, the seller is practically sure that $A$ will occur. On the other hand, $\mathrm{s} /$ he will find no rational buyer for such a price: in fact, the buyer should pay 1 to receive at most 1 if $A$ occurs, 0 otherwise. After conditioning, $\bar{E}_{P M M}(A \mid B)<1$ iff $P_{0}(A \mid B)<\frac{1}{1+\delta_{B}}=\bar{t}_{B} \leq \bar{t}_{\Omega}$. When $\bar{t}_{B}<\bar{t}_{\Omega}$, the seller may have the chance to negotiate $A$, but not $A \mid B$, for some events $A$.

Analogously, a subject 'buying' $A(A \mid B)$ will be unwilling to do so when $\underline{P}_{P M M}(A)=0$ (when $\underline{E}_{P M M}(A \mid B)=0$ ), which happens iff $P_{0}(A) \leq \frac{\delta}{1+\delta}=\underline{t}_{\Omega}$ (iff $\left.P_{0}(A \mid B) \leq \frac{\delta_{B}}{1+\delta_{B}}=\frac{\delta}{\underline{P}_{P M M}(B)+\delta}=\underline{t}_{B}\right)$. Here $\underline{t}_{B} \geq \underline{t}_{\Omega}$, and again conditioning makes the buyer more cautious, see also Fig. 1 .

1)

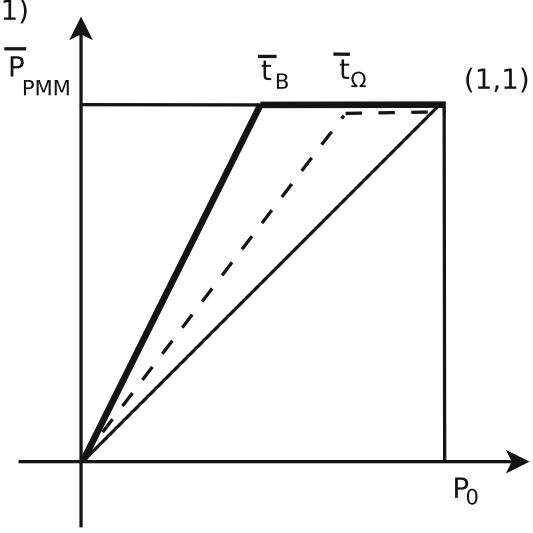

2)

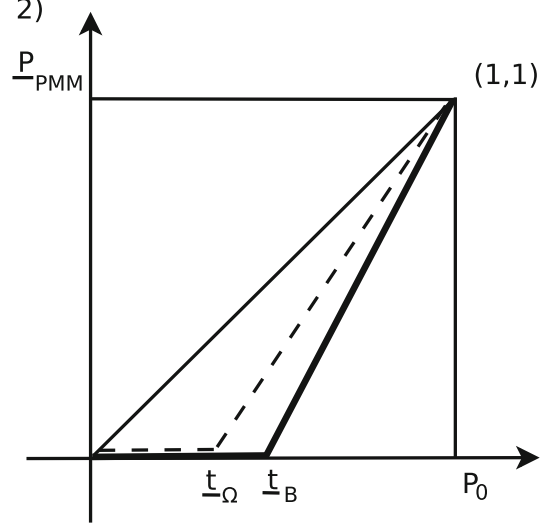

Fig. 1. 1) Plot of $\bar{P}_{P M M}$ against $P_{0}$ before (dashed bold line) and after (continuous bold line) conditioning. 2) The same for $\underline{P}_{P M M}$ against $P_{0}$.

\section{Dilation with Coherent Nearly-Linear Models}

Given a coherent NL model on $\mathcal{A}(\mathbb{P}), B \in \mathcal{A}(\mathbb{P}) \backslash\{\emptyset\}$ and an event $A \in \mathcal{A}(\mathbb{P})$, if we compute $\underline{E}(A \mid B), \bar{E}(A \mid B)$ it may happen that

$$
\underline{E}(A \mid B) \leq \underline{P}(A) \leq \bar{P}(A) \leq \bar{E}(A \mid B) .
$$

When Eq. (24) holds, the imprecision of our evaluation on $A$ increases, or at least does not decrease, after assuming $B$ true. Equation (24) is a condition 
preliminary to dilation, which we shall discuss later on. Next, we investigate when (24) holds.

Preliminarily, say that (24) occurs trivially when it holds and its three inequalities are equalities, and that $A$ is an extreme event if either $\underline{P}(A)=$ $\bar{P}(A)=0$ or $\underline{P}(A)=\bar{P}(A)=1$. When referring to extreme events in the sequel, we shall rule out $\emptyset, \Omega$ (for which no inference is needed).

Next, we investigate if Eq. (24) obtains when: $\underline{P}(B)=0$ (Lemma 2$) ; \underline{P}(B)>$ 0 and $A$ is either an extreme event (Lemma 3 ) or non-extreme (Proposition 7 ).

Lemma 2. Let $\underline{P}(B)=0$. Then,

$\left(a_{0}\right)$ If $B \nRightarrow A$ and $B \nRightarrow A^{\mathrm{c}},(24)$ applies.

$\left(b_{0}\right)$ If $B \Rightarrow A$, (24) occurs, trivially, iff $\underline{P}(A)=\bar{P}(A)=1$.

$\left(c_{0}\right)$ If $B \Rightarrow A^{\mathrm{c}},(24)$ occurs, trivially, iff $\underline{P}(A)=\bar{P}(A)=0$.

Proof. As for $\left(a_{0}\right)$, (24) holds because $\underline{E}(A \mid B)=0, \bar{E}(A \mid B)=1$ by Proposition 3 . By the same Proposition $3,\left(b_{0}\right)$ and $\left(c_{0}\right)$ follow easily.

Let us suppose now that $\underline{P}(B)>0$. From (15), (16), and since $\bar{E}(\cdot \mid B) \geq \underline{E}(\cdot \mid B)$, the following alternatives may arise:

(a) $\bar{P}(A \wedge B)=0$.

Correspondingly, $\bar{E}(A \mid B)=\underline{E}(A \mid B)=0$.

(b) $\left.\underline{P}\left(A^{\mathrm{c}} \wedge B\right), \bar{P}(A \wedge B) \in\right] 0,1[; \underline{P}(A \wedge B)=0$.

Here $\bar{E}(A \mid B) \in] 0,1[, \underline{E}(A \mid B)=0$.

(c) $\left.\underline{P}\left(A^{\mathrm{c}} \wedge B\right), \bar{P}(A \wedge B) \in\right] 0,1\left[; \underline{P}(A \wedge B), \bar{P}\left(A^{\mathrm{c}} \wedge B\right) \in\right] 0,1[$.

Then, $\bar{E}(A \mid B), \underline{E}(A \mid B) \in] 0,1[$.

(d) $\left.\underline{P}\left(A^{\mathrm{c}} \wedge B\right)=0 ; \underline{P}(A \wedge B), \bar{P}\left(A^{\mathrm{c}} \wedge B\right) \in\right] 0,1[$.

Then, $\underline{E}(A \mid B) \in] 0,1[, \bar{E}(A \mid B)=1$.

(e) $\bar{P}\left(A^{\mathrm{c}} \wedge B\right)=0$.

Correspondingly, $\bar{E}(A \mid B)=\underline{E}(A \mid B)=1$.

(f) $\underline{P}\left(A^{\mathrm{c}} \wedge B\right)=\underline{P}(A \wedge B)=0$.

Here $\underline{E}(A \mid B)=0, \bar{E}(A \mid B)=1$.

Lemma 3. If $\underline{P}(B)>0$ and $A$ is an extreme event, then (24) occurs trivially.

Proof. If $\bar{P}(A)=\underline{P}(A)=0$, then $\bar{P}(A \wedge B)=0$ by monotonicity, and from (a) above $\bar{E}(A \mid B)=\underline{E}(A \mid B)=0$ too. If $\bar{P}(A)=\underline{P}(A)=1$, then $\bar{P}\left(A^{\mathrm{c}}\right)=\underline{P}\left(A^{\mathrm{c}}\right)=$ 0 , hence $\bar{P}\left(A^{\mathrm{c}} \wedge \bar{B}\right)=0$ and $\bar{E}(A \mid B)=\underline{E}(A \mid \bar{B})=1$ from (e) above.

We still have to establish whether (24) holds assuming that $\underline{P}(B)>0$ and $A$ is a non-extreme event. Trivially, $(24)$ holds in case $(f)$, while it does not in cases $(a),(e)$. To see what happens in the remaining instances, let us prove that

Proposition 6. Given a coherent $N L$ model $(\underline{P}, \bar{P})$, let $B \in \mathcal{A}(\mathbb{P}), \underline{P}(B)>0$, and $A \in \mathcal{A}(\mathbb{P})$, A non-extreme.

(i) If $\left.\underline{P}(A \wedge B), \bar{P}\left(A^{\mathrm{c}} \wedge B\right) \in\right] 0,1[$, then

$$
\underline{E}(A \mid B) \leq \underline{P}(A) \text { iff either } P_{0}\left(B^{\mathrm{c}}\right)=0 \text { or } \underline{P}(A) \leq P_{0}\left(A \mid B^{\mathrm{c}}\right) .
$$


(ii) If $\left.\underline{P}\left(A^{\mathrm{c}} \wedge B\right), \bar{P}(A \wedge B) \in\right] 0,1[$, then

$$
\bar{E}(A \mid B) \geq \bar{P}(A) \text { iff either } P_{0}\left(B^{\mathrm{c}}\right)=0 \text { or } \bar{P}(A) \geq P_{0}\left(A \mid B^{\mathrm{c}}\right) .
$$

Proof. (i) By monotonicity, $\underline{P}(A) \geq \underline{P}(A \wedge B)>0$, and $\underline{P}(A)<1$ because $\underline{P}(A) \leq \underline{P}\left(A \vee B^{\mathrm{c}}\right)<1$, the latter inequality holding since (by conjugacy) $\left.\underline{P}\left(A \vee B^{\mathrm{c}}\right) \in\right] 0,1\left[\right.$ iff $\left.\bar{P}\left(A^{\mathrm{c}} \wedge B\right) \in\right] 0,1[$. Which is assumed to be true. Therefore, by (5), $\underline{P}(A)=b P_{0}(A)+a$, while $\underline{E}(A \mid B)$ is given by the second line in (16). Hence,

$$
\underline{E}(A \mid B) \leq \underline{P}(A) \text { iff } \frac{b P_{0}(A \wedge B)+a}{b P_{0}(B)+1-b} \leq b P_{0}(A)+a .
$$

With some algebraic manipulations on the right-hand side of (25), noting that by assumption and (2), (5), (6), (7) we have that $0<\underline{P}(B) \leq \bar{P}\left(A^{\mathrm{c}} \wedge B\right)+$ $\underline{P}(A \wedge B)=b P_{0}(B)+1-b$, we obtain

$$
\begin{aligned}
& \frac{b P_{0}(A \wedge B)+a}{b P_{0}(B)+1-b} \leq b P_{0}(A)+a \text { iff } \\
& b P_{0}(A \wedge B)+a \leq b^{2} P_{0}(A)\left(-P_{0}\left(B^{\mathrm{c}}\right)\right)+a b\left(-P_{0}\left(B^{\mathrm{c}}\right)\right)+b P_{0}(A)+a \text { iff } \\
& P_{0}\left(B^{\mathrm{c}}\right)\left(b P_{0}(A)+a\right) \leq P_{0}(A)-P_{0}(A \wedge B)=P_{0}\left(A \wedge B^{\mathrm{c}}\right) \text { iff } \\
& P_{0}\left(B^{\mathrm{c}}\right)=0 \text { or }\left(P_{0}\left(B^{\mathrm{c}}\right)>0 \text { and }\right) b P_{0}(A)+a \leq \frac{P_{0}\left(A \wedge B^{\mathrm{c}}\right)}{P_{0}\left(B^{\mathrm{c}}\right)}=P_{0}\left(A \mid B^{\mathrm{c}}\right),
\end{aligned}
$$

which proves $(i)$.

(ii) It can be obtained in a very similar way or directly by conjugacy.

From Proposition 6, and recalling (15), (16), it follows straightforwardly that

Proposition 7. Let $(\underline{P}, \bar{P})$ be a coherent $N L$ model on $\mathcal{A}(\mathbb{P}), B \in \mathcal{A}(\mathbb{P})$, $\underline{P}(B)>0$. For $A \in \mathcal{A}(\mathbb{P})$, A non-extreme, Eq. (24) holds iff

$$
\underline{P}\left(A^{\mathrm{c}} \wedge B\right)=\underline{P}(A \wedge B)=0 \text { or } P_{0}\left(B^{\mathrm{c}}\right)=0 \text { or } \underline{P}(A) \leq P_{0}\left(A \mid B^{\mathrm{c}}\right) \leq \bar{P}(A) .
$$

The left (right) inequality does not apply if $\underline{E}(A \mid B)=0$ (if $\bar{E}(A \mid B)=1$ ).

The previous results pave the way to considerations on dilation with NL models. When discussing dilation $[2,7]$, we consider a partition $\mathcal{B}$ of non-impossible events and say that (weak) dilation occurs, with respect to $A$ and $\mathcal{B}$, when

$$
\underline{E}(A \mid B) \leq \underline{P}(A) \leq \bar{P}(A) \leq \bar{E}(A \mid B), \forall B \in \mathcal{B} .
$$

Recall that an event $A$ is logically non-independent of a partition $\mathcal{B}$ iff $\exists B \in$ $\mathcal{B} \backslash\{\emptyset\}$ such that either $B \Rightarrow A$ or $B \Rightarrow A^{\mathrm{c}}$, logically independent of $\mathcal{B}$ otherwise.

We can now introduce several results concerning dilation.

Proposition 8. Let $(\underline{P}, \bar{P})$ be a coherent $N L$ model on $\mathcal{A}(\mathbb{P}), A \in \mathcal{A}(\mathbb{P}), \mathcal{B} \subset$ $\mathcal{A}(\mathbb{P}) \backslash\{\emptyset\}$ a partition.

(j) If $A$ is a non-extreme event logically non-independent of $\mathcal{B}$, dilation does not occur. 
(jj) If $A$ is an extreme event logically independent of $\mathcal{B}$, dilation occurs.

Proof. (j) Take $B \in \mathcal{B}$ such that either $B \Rightarrow A$ or $B \Rightarrow A^{\mathrm{c}}$. If $\underline{P}(B)=0,(24)$ does not occur by Lemma 2, either $\left(b_{0}\right)$ or $\left(c_{0}\right)$. If $\underline{P}(B)>0$, because of the logical non-independence hypothesis, either $A \wedge B=\emptyset$ or $A^{\mathrm{c}} \wedge B=\emptyset$, hence either $B=A^{\mathrm{c}} \wedge B$ or $B=A \wedge B$, respectively. This rules out case $(f)$, as assuming it would imply here $\underline{P}(B)=0$. Actually, recalling that $A$ is nonextreme, case (a) or (e) applies, and (24) does not occur. Thus, the dilation condition (27) is not satisfied.

(jj) Follows from Lemma $2,\left(a_{0}\right)$ when $\underline{P}(B)=0$, from Lemma 3 otherwise.

Dilation is characterised for a non-extreme event $A$, logically independent of $\mathcal{B}$, as follows:

Proposition 9. Given a coherent $N L$ model $(\underline{P}, \bar{P})$ on $\mathcal{A}(\mathbb{P})$, a partition $\mathcal{B} \subset$ $\mathcal{A}(\mathbb{P}) \backslash\{\emptyset\}$ and $A \in \mathcal{A}(\mathbb{P})$ non-extreme and logically independent of $\mathcal{B}$, dilation occurs (w.r.t. $A, \mathcal{B})$ iff, $\forall B \in \mathcal{B}$ such that $\underline{P}(B)>0$, (26) holds, where the left (right) inequality does not apply if $\underline{E}(A \mid B)=0$ (if $\bar{E}(A \mid B)=1$ ).

Proof. We need not consider those $B \in \mathcal{B}$ such that $\underline{P}(B)=0$, if any: by Lemma 2, $\left(a_{0}\right)$ they ensure (24). For the others apply Proposition 7.

We derive now an interesting sufficient condition for dilation with a VBM, extending an analogous property of a PMM [7, Corollary 2].

Proposition 10. Dilation for a non-extreme event A, logically independent of partition $\mathcal{B}$, occurs in a $V B M$ if

$$
P_{0}(A \wedge B)=P_{0}(A) \cdot P_{0}(B), \forall B \in \mathcal{B} .
$$

Proof. If $\underline{P}(B)=0$, apply Lemma 2, $\left(a_{0}\right)$. Otherwise, by Proposition 7 , we have to check that, when $P_{0}\left(B^{\mathrm{c}}\right)>0, \underline{P}(A) \leq P_{0}\left(A \mid B^{\mathrm{c}}\right) \leq \bar{P}(A)$ holds. Now, if $\underline{P}(B)>0$, then necessarily by $(5) b P_{0}(B)+a>0$, hence $P_{0}(B)>0$, because $a \leq 0$ in a VBM. Further, if $(28)$ holds, then $P_{0}\left(A \wedge B^{\mathrm{c}}\right)=P_{0}(A) \cdot P_{0}\left(B^{\mathrm{c}}\right)$, hence for those $B \in \mathcal{B}$ such that $P_{0}\left(B^{\mathrm{c}}\right)>0$, also $P_{0}\left(A \mid B^{\mathrm{c}}\right)=P_{0}(A)$. Thus, the condition to check boils down to $\underline{P}(A) \leq P_{0}(A) \leq \bar{P}(A)$, which always applies for a VBM.

Note that dilation occurs if event $A$ in Proposition 10 is $P_{0}$-non-correlated with any $B \in \mathcal{B}$.

\section{Conclusions}

Among coherent NL models, VBMs ensure the property of being stable with conditioning, as also do several known submodels of theirs, such as the PMM. This implies also that results found in [5] on natural extensions of (unconditional) VBMs to gambles still apply here to conditional gambles $X \mid B$ defined on the conditional partition $\mathbb{P} \mid B=\{\omega \mid B: \omega \in \mathbb{P}\}$. By contrast, those HBMs that 
are coherent are generally not stable, thus these models confirm their weaker properties, in comparison with VBMs, already pointed out, from other perspectives, in [1]. Concerning dilation of $A$ w.r.t. partition $\mathcal{B}$, we have seen that it may depend on more conditions, such as whether $A$ is extreme or not, or it is logically independent of $\mathcal{B}$ or not, and we supplied several results. In future work, we plan to study the regular extension [10, Appendix J] of coherent NL models, determining how its being less conservative than the natural extension may limit extreme evaluations, as well as dilation. Concepts related to dilation and not presented here are also discussed in [2] for the $\varepsilon$-contamination and the Total Variation models, among others. The assumptions in [2] are usually less general than the present framework. The role of these notions within NL models is still to be investigated.

Acknowledgments. We are grateful to the referees for their stimulating comments and suggestions. We acknowledge partial support by the FRA2018 grant 'Uncertainty Modelling: Mathematical Methods and Applications'.

\section{References}

1. Corsato, C., Pelessoni, R., Vicig, P.: Nearly-Linear uncertainty measures. International Journal of Approximate Reasoning 114, 1-28 (2019). https://doi.org/10. 1016/j.ijar.2019.08.001

2. Herron, T., Seidenfeld, T., Wasserman, L.: Divisive Conditioning: Further Results on Dilation. Philosophy of Science 64(3), 411-444 (1997). https://doi.org/10.1086/ 392559

3. Levin, D.A., Peres, Y.: Markov chains and mixing times. American Mathematical Society, Providence, R.I (2017). https://doi.org/10.1090/mbk/107

4. Montes, I., Miranda, E., Destercke, S.: Pari-mutuel probabilities as an uncertainty model. Information Sciences 481, 550-573 (2019). https://doi.org/10.1016/j.ins. 2019.01.005

5. Pelessoni, R., Vicig, P., Corsato, C.: Extending Nearly-Linear Models. In: De Bock, J., de Cooman, G., de Campos, C.P., Quaeghebeur, E., Wheeler, G. (eds.) PMLR, vol. 103, pp. 82-90 (2019)

6. Pelessoni, R., Vicig, P.: Williams coherence and beyond. International Journal of Approximate Reasoning 50(4), 612-626 (2009). https://doi.org/10.1016/j.ijar. 2008.10.002

7. Pelessoni, R., Vicig, P., Zaffalon, M.: Inference and risk measurement with the parimutuel model. International Journal of Approximate Reasoning 51(9), 1145-1158 (2010). https://doi.org/10.1016/j.ijar.2010.08.005

8. Troffaes, M.C.M., de Cooman, G.: Lower previsions. Wiley series in probability and statistics, Wiley, Chichester, West Sussex, United Kingdom (2014)

9. Walley, P.: Coherent Lower (and upper) Probabilities. Technical Report 22, Department of Statistics, University of Warwick, Coventry (1981)

10. Walley, P.: Statistical reasoning with imprecise probabilities. No. 42 in Monographs on statistics and applied probability, Chapman and Hall, London; New York (1991)

11. Williams, P.: Notes on conditional previsions. International Journal of Approximate Reasoning 44(3), 366-383 (2007). https://doi.org/10.1016/j.ijar.2006.07.019 